\title{
Generalized normal derivatives of principal functions
}

\author{
By \\ Mineko Watanabe
}

(Received May 22, 1971)

\section{Introduction}

The notion of normal operators acting into harmonic functions on a boundary neighborhood of an open Riemann surface was introduced by L. Sario, in order to construct harmonic functions with a finite number of given singularities and prescribed modes of behavior near the ideal boundary. Among those functions, especially as for the principal functions constructed by the principal operators, many interesting properties were proved.

On a compact bordered surface, for a finite number of given singularities $s$ with vanishing flux, $L_{0}$-principal function is characterized as the uniquely determined function with the $s$ whose normal derivative along the border vanishes identically, and $(P) L_{1}$-principal function with the $s$, denoting by $P$ a regular partition of the boundary, is characterized as the uniquely determined function which is constant on each part of the $P$ and whose flux over each part of the $P$ vanishes. The problems characterizing principal functions on arbitrary Riemann surfaces by these boundary behaviors under compactifications have been investigated by several authors, and cited in Sario-Oikawa [9] as open problems. In the present paper we use the notion of generalized normal derivatives to study the boundary properties of principal functions under compactifications. 
A generalized normal derivative was first defined by Doob [3] on the Martin compactification, for a function which is identical, except for some compact set, with sum of a $B L D$ harmonic function and a potential, as a function of class $L^{*}\left(\Delta_{M}\right)$ which contains all square integrable functions with respect to the harmonic measure on the Martin boundary $\Delta_{M}$. Constantinescu-Cornea [2] defined a generalized normal derivative as a signed measure on the Kuramochi boundary. Maeda [5] defined it for functions harmonic and Dirichlet integrable in boundary neighborhoods on resolutive compactifications. We use here the definitions given by Constantinescu-Cornea [2] and Maeda [5].

In $\S 1$, we consider $H M$-functions which form an important subspace of the space of $H D$-functions. It has been known that the set of $H D$-functions which have generalized normal derivatives is dense in the $H D$ by Dirichlet norms, identifying functions whose differences are constant. We shall show that the set of $H M$-functions which have generalized normal derivatives is also dense in the subspace $H M$ (Theorem 1). In $\S 2$ we generalize the definition of generalized normal derivatives given by Constantinescu-Cornea [2] to functions harmonic and Dirichlet integrable in some boundary neighborhood. We shall study about $(P) L_{1}$-principal functions only for restricted case, using the results obtained in $\S 1$. In $\S 3$ we shall concern $L_{0}$-principal functions. We characterize there $L_{0}$-principal functions in terms of generalized normal derivatives and obtain some related results including another formulation of the operator $L_{0}$ (Theorem 6).

The author expreses her hearty thanks to Professors Y. Kusunoki and K. Oikawa for their kind remarks in this research.

\section{§ Generalized normal derivatives of $\boldsymbol{H} M$-functions}

1. Throughout the present paper, we assume that a Riemann surface $R$ is hyperbolic, because all results are trivial in the case of parabolic Riemann surface, and a compactification $R^{*}$ is the Kuramochi compactification of $R$. We denote by $\Delta$ the Kuramochi boundary $R^{*}-R$. Let us denote by $D(R)$ the class of Dirichlet functions on $R$, 
by $H D(R)$ the class of harmonic functions in $D(R)$, and by $K D(R)$ the subclass of $H D(R)$ consisting of functions whose conjugates are semiexact on $R$. We identify the functions in $D(R)$ whose differences are quasi-everywhere constant on $R$. Then $D(R)$ is a Hilbert space with inner products given by Dirichlet integrals. We have an orthogonal decomposition

$$
D(R)=H D(R) \oplus \bar{C}_{0}^{\infty}(R),
$$

where $C_{0}^{\infty}(R)$ denotes a class of infinitely differentiable functions with compact supports in $R$, and $\bar{C}_{0}^{\infty}(R)$ is the closure of $C_{0}^{\infty}(R)$. We denote by $H M(R)$ the orthogonal complement of $K D(R)$ in $H D(R)$, that is

$$
H D(R)=K D(R) \oplus H M(R) .
$$

Using the notations in Ahlfors-Sario [1] for classes of differentials, a function $u$ is of class $H M(R)$ if and only if it is real and $d u$ belongs to $\Gamma_{h m}$, and $u$ is of class $K D(R)$ if and only if it is real and $d u$ belongs to $\Gamma_{h e} \cap \Gamma_{h s e}^{*}$.

According to Constantinescu-Cornea [2], we say that a function $u \in H D(R)$ has a generalized normal derivative $\mu$ on $\Delta$ if there is a signed measure $\mu$ on $\Delta$ satisfying the following condition; for any function $h \in D(R)$ we have

$$
<d u, d h>_{R}=\iint_{R} d u \wedge^{*} d h=\int_{\Delta} h d \mu
$$

denoting by the same $h$ the quasi-continuous extension of $h$ to $R^{*}$. Let $\mathfrak{N}$ be the class of functions of $H D(R)$ which have generalized normal derivatives on $\Delta$. Then $\mathfrak{N}$ is dense in $H D(R)$ ([2]), but we don't know whether $\mathfrak{R} \cap K D(R)$ is dense in $K D(R)$.

2. We shall now take up the space $H M(R)$. Let $\left\{R_{n}\right\}_{n}$ be a canonical exhaustion of $R$ (Ahlfors-Sario [1]), and $\left\{\gamma_{k}\right\}_{k}$ a basis to all dividing cycles on $R$. For any fixed $\gamma_{k}$, we choose $n$ so large that $R_{n}$

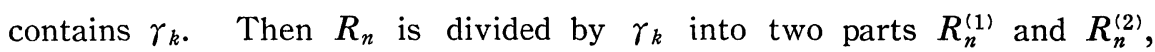


and the boundary $\partial R_{n}$ of $R_{n}$ is also divided into $\Gamma_{n}^{(1)}=\partial R_{n}^{(1)} \cap \partial R_{n}$ and $\Gamma_{n}^{(2)}=\partial R_{n}^{(2)} \cap \partial R_{n}$. We take a harmonic function $u_{k}^{(n)}$ on $R_{n}$ which is 1 on $\Gamma_{n}^{(1)}$ and 0 on $\Gamma_{n}^{(2)}$. The limit function $u_{k}$ of $\left\{u_{k}^{(n)}\right\}$ as $n$ tends to $\infty$ is an element of $H D(R)$, and the $H M(R)$ is generated by those $u_{k}(k=1,2, \ldots)$ (Ahlfors-Sario $[1]$ and Kusunoki $[4]$ ).

Theorem 1. The subset $\mathfrak{N} \cap H M(R)$ is dense in $H M(R)$.

Proof. In order to prove this Theorem, it is sufficient to show that each $u_{k}$ has a generalized normal derivative on $\Delta$.

We choose a canonical exhaustion $\left\{R_{n}\right\}$ so that each component of $\partial R_{n}$, and therefore each component of $\Gamma_{n}^{(1)}$ and $\Gamma_{n}^{(2)}$, is piecewise smooth. Hereafter we pick up one $\gamma_{k}$, and denote it by $\gamma$ and the corresponding $u_{k}$ by $u$ for simplicity. For $\gamma \subset R_{n}$ we introduce measures $\mu_{n}$ on $R^{*}$ as follows:

$$
d \mu_{n}=\left\{\begin{array}{lll}
* d u^{(n)}=-\frac{\partial u^{(n)}}{\partial n} d s & \text { on } & \partial R_{n} \\
0 & \text { on } & R^{*}-\partial R_{n}
\end{array}\right.
$$

where derivatives are construed as inner normal derivatives relative to $R_{n}$. Observing

$$
0=<d u^{(n)}, d 1>_{R_{n}}=\int_{\Gamma_{n}^{(1)}} * d u^{(n)}+\int_{\Gamma_{n}^{(2)}} * d u^{(n)}
$$

we get that

$$
\int_{R^{*}}\left|d \mu_{n}\right|=2 \int_{\Gamma_{n}^{(1)}} * d u^{(n)}
$$

Therefore, by the facts that

$$
\left\|d u^{(n)}\right\|_{R_{n}}^{2}=<d u^{(n)}, d u^{(n)}>_{R_{n}}=\int_{\Gamma_{n}^{(1)}} * d u^{(n)}
$$

and 


$$
\lim _{n \rightarrow \infty}\left\|d u^{(n)}\right\|_{R_{n}}^{2}=\|d u\|_{R}^{2}<\infty,
$$

we obtain that the sequence of the total variations of signed measures $\left\{\mu_{n}\right\}$ are uniformly bounded. Thus, there exists a subsequence $\left\{\mu_{n_{j}}\right\}$ of $\left\{\mu_{n}\right\}$ which converges to a measure $\mu$, and the support of $\mu$ is contained in $\Delta$.

We show that the sequence $\left\{\mu_{n}\right\}$ converges to the $\mu$. For any subsequence of $\left\{\mu_{n}\right\}$, there exists a convergent subsequence $\left\{\mu_{n_{k}}\right\}$. Let the limit of $\left\{\mu_{n_{k}}\right\}$ be $\tilde{\mu}$, and consider the following class of functions on $\Delta$;

$$
\mathfrak{M}=\left\{f \text { on } \Delta \mid \int_{\Delta} f d \mu=\int_{\Delta} f d \tilde{\mu}\right\}
$$

The $\mathfrak{M}$ is a monotone class. For any function $h \in D(R) \cap C\left(R^{*}\right)$, where $C\left(R^{*}\right)$ denotes the class of functions which are continuous on $R^{*}$, we have

$$
\lim _{j \rightarrow \infty}<d u^{\left(n_{j}\right)}, d h>_{R_{n_{j}}}=\lim _{j \rightarrow \infty} \int_{R^{*}} h d \mu_{n_{j}}=\int_{\Delta} h d \mu
$$

and

$$
\lim _{k \rightarrow \infty}<d u^{\left(n_{k}\right)}, d h>_{R_{n_{k}}}=\lim _{k \rightarrow \infty} \int_{R^{*}} h d \mu_{n_{k}}=\int_{\Delta} h d \tilde{\mu} .
$$

On the other hand, for any function $h \in D(R)$, we have

$$
<d u^{(n)}, d h>_{R_{n}}=\int_{\partial R_{n}} h^{*} d u^{(n)}
$$

and

$$
\lim _{n \rightarrow \infty}<d u^{(n)}, d h>_{R_{n}}=<d u, d h>_{R} .
$$

Therefore it holds that

$$
\int_{\Delta} h d \mu=\int_{\Delta} h d \tilde{\mu}
$$

for $h \in D(R) \cap C\left(R^{*}\right)$. We denote by $C_{D}(\Delta)$ the class of restrictions of functions $\in D(R) \cap C\left(R^{*}\right)$ to $\Delta$. We obtain by (4) that the monotone 
hull of $C_{D}(\Delta)$ is contained in $\mathfrak{M}$. The $C_{D}(\Delta)$ is dense in $C(\Delta)$ in the uniform convergence topology, where $C(\Delta)$ denotes the class of continuous functions on $\Delta$, and therefore $C(\Delta)$ is contained in the monotone hull of $C_{D}(\Delta)$. Hence, any continuous function $f$ on $\Delta$ belongs to $\mathfrak{M}$, that is

$$
\int_{\Delta} f d \mu=\int_{\Delta} f d \tilde{\mu}
$$

and we conclude that the sequence of measures $\left\{\mu_{n}\right\}$ converges to the $\mu$ which is a measure on $\Delta$.

In order to assert that this $\mu$ is a generalized normal derviative of the $u$, we should show that (2) holds for any function of class $D(R)$. If $h$ belongs to $D(R) \cap C\left(R^{*}\right)$, (2) is immediate, since

$$
\begin{aligned}
<d u, d h>_{R} & =\lim _{n \rightarrow \infty}<d u^{(n)}, d h>_{R_{n}} \\
& =\lim _{n \rightarrow \infty} \int_{R^{*}} h d \mu_{n}=\int_{\Delta} h d \mu .
\end{aligned}
$$

Assume that $h \in D(R)-D(R) \cap C\left(R^{*}\right)$. Then, for any positive number $\varepsilon$, we can choose an open set $G$ with Kuramochi capacity less than $\varepsilon$ so that the restriciton of $h$ to $R^{*}-G$ is continuous. The Kuramochi capacity of an open set $G$ is the number

$$
\widetilde{C}(G)=\sup _{K \subset G} \tilde{C}(K)
$$

where $K$ are compact sets contained in $G$, and the Kuramochi capacity of a compact set $K$ is the number

$$
\tilde{C}(K)=\sup _{\nu} \nu(K)
$$

where $\nu$ are the canonical measures such that $\tilde{p}^{\nu} \leqslant 1$. (ConstantinescuCornea [2]). Here $\tilde{p}^{\nu}$ denotes the potential

$$
\tilde{p}^{\nu}(a)=\int \tilde{g}_{a} d \nu
$$


using the function $\tilde{g}_{a}$ defined in p. 159 of $[2]$. We fix a point $a \in$ $R_{1}-\bar{G}$, then the harmonic measure $\omega_{a}^{R_{n}}$ of $R_{n}$ with respect to $a$ as well as the harmonic measure $\omega_{a}^{R, R^{*}}=\omega_{a}$ on $\Delta$ is canonical (cf. [2]). We denote by $g_{a}^{R_{n}}$ the Green function of $R_{n}$ with a pole at $a$, and let

$$
\mu_{n}^{+}=\max \left(0, \mu_{n}\right), \quad \mu_{n}^{-}=\max \left(0,-\mu_{n}\right)
$$

Then, we have

$$
d \mu_{n}^{-} \leqslant \frac{\partial g_{a}^{R_{n}}}{\partial n} d s=2 \pi d \omega_{a}^{R_{n}}
$$

because $0 \leqslant u^{(n)} \leqslant g_{a}^{R_{n}}$ in some neighborhood of $\Gamma_{n}^{(2)}$ in $R_{n}$, and

$$
\omega_{a}^{R_{n}} \leqslant \tilde{\omega}_{a}^{R_{n}}
$$

On the other hand, it holds that

$$
\begin{aligned}
& \tilde{p}^{\tilde{\omega}_{a}^{R}}(b)=\int \tilde{g}_{b} d \tilde{\omega}_{a}^{R_{n}} \\
& =\left(\tilde{g}_{b}\right) \widetilde{R-R_{n}}(a)=\left(\tilde{g}_{a}\right) \widetilde{R-R_{n}}(b) .
\end{aligned}
$$

As for notations $\widetilde{\omega}_{a}^{R_{n}},\left(\tilde{\mathrm{g}}_{b}\right)_{R-R_{n}}$ and the last equation, refer to pages 158, 164 and 166 of [2]. Therefore, if we put

$$
\lambda_{n}=\sup _{b \in K}\left(\tilde{g}_{b}\right)_{R-R_{n}}^{\sim}(a) \text {, and } \lambda=\sup _{n} \lambda_{n},
$$

the number $\lambda$ is finite, and we have

$$
\tilde{p}^{\omega_{a}^{R} / \lambda} \leqslant \tilde{p}^{\tilde{\omega}_{a}^{R} / \lambda} \leqslant 1 .
$$

Hence, by (5), (6) and (8) we get

$$
\omega_{a}^{R_{n}}(K) \leqslant \lambda \widetilde{c}(K)<\lambda \varepsilon
$$

and by (7) we get

$$
\mu_{n}^{-}(G) \leqslant 2 \pi \lambda \varepsilon
$$


for any natural number $n$. For $\mu_{n}^{+}$we get the same inequality

$$
\mu_{n}^{+}(G) \leqslant 2 \pi \lambda \varepsilon
$$

because $0 \leqslant 1-u^{(n)} \leqslant g_{a}^{R_{n}}$ in a neighbouhood of $\Gamma_{n}^{(1)}$ in $R_{n}$. By (9) and $\left(9^{\prime}\right)$, we readily obtain

$$
\begin{aligned}
<d u, d h>_{R} & =\lim _{n \rightarrow \infty}<d u^{(n)}, d h>_{R_{n}} \\
& =\lim _{n \rightarrow \infty} \int_{R^{*}} h d \mu_{n}=\int_{R^{*}} h d \mu .
\end{aligned}
$$

(For details of the proof, see the note at the end of the paper).

3. Let $P$ be a regular partition of the ideal boundary. We denote by $I$ and $Q$ the identity partition and the canonical partition respectively. We denote by $H M_{P}(R)$ the class of functions which is spanned by the functions $u_{P_{k}}$ corresponding to $P$-dividing cycles $\gamma_{P_{k}}$. The fact that $u_{P_{k}}$ has a generalized normal derivative on $\Delta$ is proved quite analogously as for $u_{k}$. Therefore we get

Corollary 1. If the number of parts of partition $P$ is finite, any function of $H M_{P}(R)$ has a generalized normal derivative on $\Delta$. In particular, if the number of boundary components of $R$ is finite, any function of $H M(R)$ has a generalized normal derivative on $\Delta$.

4. Maeda [5] introduced the notions of $D$-normal compactifications and regular compactifications. We say that a resolutive compactification is $D$-normal if all restrictions to the boundary of the extensions of functions $\in H D(R)$ are resolutive, and regular if the $C_{D}\left(\Delta^{\prime}\right)$ is dense in the $C\left(\Delta^{\prime}\right)$ in the uniform convergence topology, where $\Delta^{\prime}$ denote the boundary on the compactification. Many familiar compactifications including those of Kuramochi, Martin, Royden and Wiener are $D$-normal, and those of Kuramochi and Royden are regular $([5])$. We denote by 
$R_{D}^{*}$ a $D$-normal compactification and by $\Delta_{D}$ the boundary on $R_{D}^{*}$. Maeda [5] defined a generalized normal derivative on any resolutive compactification for a function $f$ which is harmonic and Dirichlet integrable in $R-K$, where $K$ is a compact set with smooth boundary. We say that a function $\varphi$ on $\Delta_{D}$ is a generalized normal derivative of $f$ in the sense of Maeda [5], if for a restriction $\psi$ to the boundary $\Delta_{D}$ on a $D$-normal compactification of any function $\in H D(R)$, we have

$$
<d f, d v_{\psi, K}>_{R-K}=-\int \varphi \psi d \omega_{a}
$$

where $v_{\psi, K}$ is the function which is identically 0 on $K$ and harmonic on $R-K$ with boundary value $\psi$ on $\Delta^{\prime}$ and 0 on $\partial K$ whenever $\psi$ is resolutive, and $\varphi \psi$ is of class $L^{1}\left(\omega_{a}\right)$.

In the proof of Theorem 1 , we have the limit $\mu$ of the sequence $\left\{\mu_{n}\right\}$ on any regular compactification. By (7) and the same inequality for positive part $\mu_{n}^{+}$of $\mu_{n}$ for any $n$, we easily get that the $\mu$ is absolutely continuous with respect to the $\omega_{a}$, and we have

$$
d \mu=-\varphi d \omega_{a}
$$

with $|\varphi| \leqslant 2 \pi$. Hence, if the compactification is $D$-normal we have $\varphi \psi \in$ $L^{1}\left(\omega_{a}\right)$ for any $\psi$ which is a restriction to $\Delta_{D}$ of a function $\in H D(R)$, and it is readily verified that

$$
<d u, d v_{\psi, K}>_{R-K}=-\int \varphi \psi d \omega_{a}
$$

holds for any $\psi$.

Thus, from the proof of Theorem 1 , we reach to

Corollary 2. On the regular and D-normal compactification, the set of functions of class $H M(R)$ which have generalized normal derivatives on $\Delta_{D}$ in the sense of Maeda [5] is dense in $H M(R)$. If the number of parts of regular partition $P$ is finite, every functions of class $H M_{P}(R)$ have generalized normal derivatives on $\Delta_{D}$. 


\section{$\S 2$ Generalized normal derivatives of $(\boldsymbol{P}) \boldsymbol{L}_{1}$-principal functions}

5. Let $\Omega$ be a canonical region with smooth boundary on $R$, and $\left\{R_{n}\right\}$ a canonical exhaustion such that $\bar{\Omega} \subset R_{1}$. The normal operators $L_{0}$ and $(P) L_{1}$ on $R_{n}-\bar{\Omega}$ are defined as follows. For a given real function $f$ of class $C^{1}$ on $\partial \Omega$ we construct $u_{0}^{(n)}=L_{0} f$ as the harmonic function in $R_{n}-\bar{\Omega}$ which has the boundary value $f$ on $\partial \Omega$ and whose normal derivative vanishes on $\partial R_{n}$. We construct $u_{P}^{(n)}=(P) L_{1} f$ as the harmonic function in $R_{n}-\bar{\Omega}$ which has the boundary value $f$ on $\partial \Omega$, and is constant on each part of $P$ and has a vanishing flux over each part of $P$ separately. Here $P$ is of course the partition of $\partial R_{n}$ induced by the original partition $P$ of the ideal boundary of $R$. The sequences of functions $\left\{u_{0}^{(n)}\right\}_{n}$ and $\left\{u_{P}^{(n)}\right\}_{n}$ converge in norm sense to the harmonic functions $u_{0}$ and $u_{P}$ in $R-\bar{\Omega}$ respectively. We define that $u_{0}=L_{0} f$ and $u_{P}=(P) L_{1} f$ in $R-\bar{\Omega}$. The operators $L_{0}$ and $(P) L_{1}$ are normal operators with respect to $R-\bar{\Omega}$. Let $E$ be a finite point set on $\Omega$ and let $s$ be given singularities at the points of $E$ with vanishing flux. Then, there exist unique functions $f_{0}$ and $f_{P}$ on $R$ with the singularities $s$ which are constructed by the operators $L_{0}$ and $(P) L_{1}$ and are called as an $L_{0}$-principal function and a $(P) L_{1}$-principal function respectively (Ahlfors-Sario [1]). On each $R_{n}$, there are the $L_{0}$-principal function $f_{0}^{(n)}$ and $(P) L_{1}$-principal function $f_{P}^{(n)}$ with the singularities $s$.

Lemma 1. (Rodin-Sario [7]) Suitably normalized sequences $\left\{f_{0}^{(n)}\right\}_{n}$ and $\left\{f_{P}^{(n)}\right\}_{n}$ converge to $f_{0}$ and $f_{P}$ uniformly on any compact set on $R$.

Lemma 2. (Watanabe $[10]$ ) The $f_{0}$ and $f_{P}$ are approximated in norm by $\left\{f_{0}^{(n)}\right\}_{n}$ and $\left\{f_{P}^{(n)}\right\}_{n}$ respectively, that is, $\lim _{n \rightarrow \infty}\left\|d\left(f_{0}-f_{0}^{(n)}\right)\right\|_{R_{n}}=0$ and $\lim _{n \rightarrow \infty}\left\|d\left(f_{P}-f_{P}^{(n)}\right)\right\|_{R_{n}}=0$.

6. Nakai-Sario [6] proved that an $(I) L_{1}$-principal function can be extended finitely continuously to the Royden compactification so that 
the extension is almost everywhere constant, and this property and the vanishing of flux over the ideal boundary characterize $(I) L_{1}$-principal functions. More generally, combining parts of Theorems 1 and 2 in Watanabe $[10]$ we get

Lemma 3. If the number of parts of regular partition $P$ is finite, $(P) L_{1}$-principal functions, which are harmonic except for a finite number of singularities with vanishing flux, are characterized by the following conditions:

i) they are Dirichlet integrable in boundary neighborhoods and have vanishing fluxes over every P-dividing cycles, and

ii) they can be extended almost everywhere (or quasi-everywhere) continuously to the compactifications so that the extensions are a.e. (or q.e.) constant on each part of the P. Here the compactifications may be those of Kuramochi, Martin, Royden, Wiener or $\mathfrak{\Omega}$-compactifications denoting by $\mathfrak{Q}$ any sublattice of HP which contains constant.

Suppose that at a finite number of points $a_{j} \in R$ with local parameters $\zeta_{j}$, there are given singularities $s$ of the form

$$
\operatorname{Re} \sum_{n=1}^{\infty} b_{n}^{(j)} \zeta_{j}^{-n}+c^{j} \log \left|\zeta_{j}\right|
$$

where the $c^{(j)}$ are real and subject to the condition $\sum_{j} c^{(j)}=0$. Then, there exists a unique $(I) L_{1}$-principal function $f_{I}$ with the $s$. We separate the $s$ into two parts: 1) the $s_{1}$ consisting of all terms of the form $\operatorname{Re} \sum b_{n}^{(j)} \zeta_{j}^{-n}$, and 2) the $s_{2}$ consisting of all logarithmic terms of the $s$. Then, it is readily seen by Lemma 3 the following

Lemma 4. The (I) $L_{1}$-principal function $f_{I}$ with the singularities $s$ is the sum

$$
f_{I}=f_{I, 1}+f_{I, 2}+\text { constant, }
$$

where $f_{I, k}$ denote the $(I) L_{1}$-principal functions with the singularities 
$s_{k}(k=1,2)$. And the $f_{I, 2}$ is the sum

$$
f_{I, 2}=\sum_{j} c^{(j)} g_{j}+\text { constant }
$$

where $g_{j}$ denote the Green functions on $R$ with poles at $a_{j}$.

7. Now we generalize the definition of generalized normal derivatives on the Kuramochi boundary given by Constantinescu-Cornea [2] for functions of class $H D(R)$, to any function which is harmonic and Dirichlet integrable in a boundary neighborhood. For a boundary neighborhood $U$, we denote by $H D(\bar{U})$ the class of functions which are harmonic and Dirichlet integrable on $\bar{U}$.

Definition. For a function $f$ of $H D(\bar{U})$, we say that $f$ has a generalized normal derivative $\mu$ on $\Delta$, if there exists a signed measure $\mu$ satisfying

$$
<d f, d h>_{U}=\int_{\Delta} h d \mu+\int_{\partial U} h^{*} d f
$$

for any $h$ of $D(R)$.

It is readily seen that this definition is independent of the choice of $U$, and for any function of class $H D(R)$, coincides with the original definition.

Lemma 5. The Green function $g_{j}$ has a generalized normal derivative $-2 \pi \omega_{a_{j}}$ on $\Delta$.

This Lemma was established as Proposition 1 in Maeda [5] according to his definition of generalized normal derivatives. We give here another proof which is analogous to that of Theorem 1.

Proof. The Green function $g_{j}$ is a limit of sequence of Green functions $\left\{g_{j}^{(n)}\right\}_{n}$, where $g_{j}^{(n)}$ denote the Green functions on $R_{n}$ with poles at $a_{j}$. The $\left\{g_{j}^{(n)}\right\}_{n}$ converges uniformly on any compact set and 
$\lim _{n \rightarrow \infty}\left\|d\left(g_{j}-g_{j}^{(n)}\right)\right\|_{R_{n}}=0$. We define non-positive measures $\mu_{j, n}=-2 \pi \omega_{a_{j}}^{R_{n}}$ as follows:

$$
d \mu_{j, n}=\left\{\begin{array}{lll}
* d g_{j}^{(n)}=-\frac{\partial g_{j}^{(n)}}{\partial n} d s & \text { on } & \partial R_{n} \\
0 & \text { on } & R^{*}-\partial R_{n} .
\end{array}\right.
$$

Because of

$$
\int_{\partial R_{n}} \frac{\partial g_{j}^{(n)}}{\partial n} d s=\int_{\partial \Omega} \frac{\partial g_{j}^{(n)}}{\partial n} d s
$$

and uniform convergence of $\left\{g_{j}^{(n)}\right\}_{n}$ on $\partial \Omega$, we get that the measures $\mu_{j, n}$ are uniformly bounded, and therefore there is a measure $\mu_{j}=-2 \pi \omega_{a_{j}}$ on $\Delta$ which is a limit of the sequence $\left\{\mu_{j, n}\right\}_{n}$. Further, for any $h \in$ $D(R)$, we can easily show that

$$
\begin{aligned}
\left.<d g_{j}, d h\right\rangle_{R-\bar{\Omega}} & =\lim _{n \rightarrow \infty}\left\langle d g_{j}^{(n)}, d h>_{R_{n}-\bar{\Omega}}\right. \\
& =\lim _{n \rightarrow \infty}\left(\int_{\partial R_{n}} h^{*} d g_{j}^{(n)}+\int_{\partial \Omega} h^{*} d g_{j}^{(n)}\right) \\
& =-2 \pi \int_{\Delta} h d \omega_{a_{j}}+\int_{\partial \Omega} h^{*} d g_{j}^{(n)}
\end{aligned}
$$

This implies that the $-2 \pi \omega_{a_{j}}$ is a generalized normal derivative on $\Delta$ of the $g_{j}$.

8. For a generalized normal derivative $\mu$ of a function of $H D(R)$, we have always $\mu(\Delta)=0$, because

$$
<d u, d 1>_{R}=\int_{\Delta} d \mu=0 .
$$

It should be remarked that for a generalized normal derivative $\mu$ of a function $f \in H D(\bar{U})$, we do not necessarily have $\mu(\Delta)=0$, but we merely assert that $\mu(\Delta)=-\int_{\partial U} * d f$.

Combining Lemmas 4 and 5 , we obtain

Theorem 2. An $(I) L_{1}$-principal function $f_{I}$ with only logarithmic 
singularities has a generalized normal derivative $\mu_{I}$ on $\Delta$ such that $\mu_{I}(\Delta)=0$.

Proof. The $\mu_{I}$ is given as a sum $-2 \pi \sum_{j} c^{(j)} \omega_{a_{j}}$ and also as a limit of $\sum_{j} c^{(j)} \mu_{j, n}$ as $n \rightarrow \infty$, using the notations in above Lemmas. The $f_{I}$ is a limit of $\left\{f_{I}^{(n)}\right\}_{n}$, where $f_{I}^{(n)}$ are the $(I) L_{1}$-principal functions on $R_{n}$ with the same singularities as the $f_{I}$, and the measures $\sum_{j} c^{(j)} \mu_{j, n}$ are equal to the measures $\mu_{I, n}$ defined as

$$
d \mu_{I, n}=\left\{\begin{array}{lll}
* d f_{I}^{(n)} & \text { on } & \partial R_{n} \\
0 & \text { on } & R^{*}-\partial R_{n} .
\end{array}\right.
$$

We have

$$
\mu_{I, n}\left(R^{*}\right)=\int_{\partial R_{n}} * d f_{I}^{(n)}=0
$$

by definition, and therefore

$$
\begin{aligned}
\mu_{I}(\Delta)=\mu_{I}\left(R^{*}\right) & =\int_{R^{*}} d \mu_{I}=\lim _{n \rightarrow \infty} \int_{R^{*}} d \mu_{I, n} \\
& =\lim _{n \rightarrow \infty} \mu_{I, n}\left(R^{*}\right)=0 .
\end{aligned}
$$

9. By Lemmas 1,2 in Watanabe $[10]$ and the definition of $(P) \Gamma_{h m}$, we can easily verify

Lemma 6. For any regular partition $P$ of the ideal boundary, $f$ is a $(P) L_{1}$-principal function if and only if it is represented as

$$
f=f_{I}+u_{P}
$$

where $f_{I}$ is an $(I) L_{1}$-principal function and $d u_{P}$ is an element of $(P) \Gamma_{h m}$ such that

$$
\int_{\gamma_{P}} * d f_{I}=-\int_{\gamma_{P}} * d u_{P}
$$


for any P-dividing cycle $\gamma_{P}$.

Theorem 3. Suppose that a regular partition $P$ consists of only a finite number of parts. Then, a $(P) L_{1}$-principal function $f_{P}$ with only logarithmic singularities has a generalized normal derivative $\mu_{P}$ on $\Delta$, and $\mu_{P}\left(\Delta^{(\alpha)}\right)=0$ for any part $\Delta^{(\alpha)}$ of the partition $P$.

Proof. The first assertion of the Theorem is gained by Theorem 2, Lemma 6 and Corollary 1. In order to show that $\mu_{P}\left(\Delta^{(\alpha)}\right)=0$, we take the partition $P_{n}$ of each $\partial R_{n}$ which is induced by the original $P$. Observing the proofs of Corollary 1 and Theorem 2, and using Lemmas 1,2 and 6 , we readily know that the $\mu_{P}$ is a limit of measures $\left\{\mu_{P, n}\right\}_{n}$, where

$$
d \mu_{P, n}=\left\{\begin{array}{lll}
{ }^{*} d f_{P}^{(n)} & \text { on } & \partial R_{n} \\
0 & \text { on } & R^{*}-\partial R_{n} .
\end{array}\right.
$$

Of course $f_{P}^{(u)}$ denotes the $(P) L_{1}$-principal function on $R_{n}$ with the same singularities as the $f_{P}$. We consider the restriction of $\mu_{P}$ to $\Delta^{(\alpha)}$ and denote it by $\mu_{P}^{(\alpha)}$. We denote by $\gamma_{n}^{(\alpha)}$ the part of $P_{n}$ which bounds the set $G_{n}^{(\alpha)}\left(R-R_{n}\right.$ carrying $\Delta^{(\alpha)}$ as a part of ideal boundary. Let $\mu_{P, n}^{(\alpha)}$ be the restriction of $\mu_{P, n}$ to $\gamma_{n}^{(\alpha)}$. If $n$ is so large that $R_{n}$ contains a basis of all $P$-dividing cycles in $R$, we have

$$
\begin{aligned}
\mu_{P, n}^{(\alpha)}\left(R^{*}\right) & =\mu_{P, n}^{(\alpha)}\left(\gamma_{n}^{(\alpha)}\right)=\mu_{P, n}\left(\gamma_{n}^{(\alpha)}\right) \\
& =\int_{\gamma_{n}^{(\alpha)}} * d f_{P}^{(n)}=0
\end{aligned}
$$

The $\mu_{P}^{(\alpha)}$ is clearly a limit of $\left\{\mu_{P, n}^{(\alpha)}\right\}_{n}$, and therefore we get

$$
\mu_{P}\left(\Delta^{(\alpha)}\right)=\mu_{P}^{(\alpha)}\left(R^{*}\right)=\lim _{n \rightarrow \infty} \mu_{P, n}^{(\alpha)}\left(R^{*}\right)=0
$$

10. It is evident that Theorems 2 and 3 are valid for generalized normal derivatives in the sense of Maeda [5] on the regular and $D$-normal compactification, because of Corollary 2 and the fact that 
Lemma 5 holds in the sense of Maeda [5].

\section{$\S 3 L_{0}$-principal functions}

11. In this section we shall study about $L_{0}$-principal functions. The following Theorem was essentially established in Sario-Oikawa [9] without using the words of generalized normal derivatives. We state it here in terms of generalized normal derivatives.

Theorem 4. A function $f$ which is harmonic except for a finite number of singularities and Dirichlet integrable in a boundary neighborhood is an $L_{0}$-principal function if and only if $f$ has a generalized normal derivative on $\Delta$ which vanishes identically.

Proof. Let $f_{0}$ be an $L_{0}$-principal function on $R$ with singularities $s$. We take a canonical region $\Omega$ carrying all the $s$, and a canonical exhaustion $\left\{R_{n}\right\}$ with smooth boundaries. Then, there are $L_{0}$-principal functions $f_{0}^{(n)}$ on $R_{n}$ with the singularities $s$. For any $h \in D(R)$, we have

$$
\begin{aligned}
\left\langle d f_{0}^{(n)}, d h>_{R_{n}-\bar{\Omega}}\right. & =\int_{\partial R_{n}} h^{*} d f_{0}^{(n)}-\int_{\partial \Omega} h^{*} d f_{0}^{(n)} \\
& =-\int_{\partial \Omega} h^{*} d f_{0}^{(n)},
\end{aligned}
$$

because $* d f_{0}^{(n)}=0$ along $\partial R_{n}$ by definition. Further we have

$$
\lim _{n \rightarrow \infty}<d f_{0}^{(n)}, d h>_{R_{n}-\bar{\Omega}}=\left\langle d f_{0}, d h>_{R-\bar{\Omega}}\right.
$$

by Lemma 2 , and

$$
\lim _{n \rightarrow \infty} \int_{\partial \Omega} h^{*} d f_{0}^{(n)}=\int_{\partial \Omega} h^{*} d f_{0}
$$

by Lemma 1 . Therefore we get

$$
<d f_{0}, d h>_{R-\bar{\Omega}}=-\int_{\partial \Omega} h^{*} d f_{0} .
$$


This implies that $f_{0}$ has a generalized normal derivative on $\Delta$ which vanishes identically.

Conversely, suppose that a harmonic function $f$ except for a finite number of singularities is Dirichlet integrable in a boundary neighorhood and has a generalized normal derivative $\mu$ on $\Delta$ such that $\mu \equiv 0$. We construct an $L_{0}$-principal function $f_{0}$ with the same singularities as $f$. The $f_{0}$ has a generalized normal derivative $\mu_{0} \equiv 0$. Consider the function $f-f_{0}$, which is of class $H D(R)$. 'The $f-f_{0}$ has a generalized normal derivative $\mu-\mu_{0}$ which vanishes identically. For any $h \in D(R)$, we have

$$
<d\left(f-f_{0}\right), d h>_{R}=\int_{\Delta} h d\left(\mu-\mu_{0}\right)=0
$$

and if we take the $f-f_{0}$ as $h$

$$
\left\|d\left(f-f_{0}\right)\right\|_{R}=0
$$

which means $f-f_{0}=$ constant on $R$, or the $f$ is an $L_{0}$-principal function.

Corollary 3. If a function $u \in H D(R)$ has a generalized normal derivative $\mu$ on $\Delta$, there exists a harmonic function $f$ whose generalized normal derivative is the same $\mu$ and which has a finite number of given singularities with vanishing flux and is Dirichlet integrable in a boundary neiborhood. Conversely, if a function $f$ harmonic except for a finite number of singularities with vanishing fux and Dirichlet integrable in a boundary neighborhood has a generalized normal derivative $\mu$ on 4 , there exists a function $u \in H D(R)$ with the generalized normal derivative $\mu$ on $4 . \quad$ Moreover, the $u$ is of class $K D(R)$ if and only if a flux of $f$ over any dividing cycle vanishes.

Proof. Let $s$ be given singularities with vanishing flux and $f_{0}$ the $L_{0}$-principal function with the $s$. Then, the following function $f$

$$
f_{0}+u=f
$$


has the same generalized normal derivative as the $u$ by Theorem 4 . The remaining parts of the Corollary follows also immediately from Theorem 4 .

12. By the same reasoning of this Corollary, we get the structure of $L_{0}$-principal functions from Theorem 2 .

Theorem 5. A function $f$ which is harmonic except for a finite number of logarithmic singularities with vanishing flux and Dirichlet integrable in a boundary neighborhood is an $L_{0}$-principal function, if and only if it is represented as

$$
f=f_{I}+u
$$

where $f_{I}$ denotes the $(I) L_{1}$-principal function with the same singularities as the $f$, and $u$ is a function $\in H D(R)$ with generalized normal derivative $-\mu_{I}$. Here we denote by $\mu_{I}$ the generalized normal derivative of the $f_{I}$.

13. The function $v_{\psi, K}$ in the equation (10) is a Dirichlet function, and the equation (12) shows that

$$
<d f_{0}, d v_{\phi, K}>_{R-\bar{\Omega}}=0,
$$

that is, the $L_{0}$-principal function $f_{0}$ has a generalized normal derivative $\varphi \equiv 0$ on $\Delta_{D}$ in the sense of Maeda [5]. Conversely, from the equation (13) we obtain (12). Indeed, any $h \in D(R)$ has a decomposition

$$
h=u_{h}+p_{h},
$$

where $u_{h} \in H D(R)$ and $p_{h} \in \bar{C}_{0}^{\infty}(R)$ by (1), and

$$
u_{h}=v_{\psi, \bar{\Omega}}+v_{h} \quad \text { on } \quad R-\Omega,
$$

where $\psi$ is a restriction of $u_{h}$ to $\Delta_{D}$ and $v_{h}$ denotes a harmonic function on $R-\Omega$ with boundary value $u_{h}$ on $\partial \Omega$ and 0 on $\Delta_{D}$. Then we have

$$
\begin{aligned}
& <d f_{0}, d h>_{R-\bar{\Omega}} \\
& \quad=<d f_{0}, d v_{\psi, \bar{\Omega}}>_{R-\bar{\Omega}}+<d f_{0}, d v_{h}>_{R-\bar{\Omega}}+<d f_{0}, d p_{h}>_{R-\bar{\Omega}}
\end{aligned}
$$




$$
=-\int_{\partial \Omega} v_{h}^{*} d f_{0}-\int_{\partial \Omega} p_{h}^{*} d f_{0}=-\int_{\partial \Omega} h^{*} d f_{0}
$$

Therefore Theorem 4, 5 and Corollary 3 are valid for generalized normal derivatives on $\Delta_{D}$ in the sense of Maeda [5].

Maeda [5] proved that the function

$$
v^{(a)}=\frac{1}{2 \pi}\left(g_{a}-g_{a_{0}}\right)+u_{a}
$$

with a fixed $a_{0} \in R$, has a generalized normal derivative zero in his sense. Here $u_{a}$ denotes the reproducing function in the $H D(R)$, that is

$$
<d u_{a}, d u>=u(a)-u\left(a_{0}\right)
$$

for any $u \in H D(R)$. Therefore we get that the $v^{(a)}$ is an $L_{0}$-principal function $f_{0}^{(a)}$ with the logarithmic poles $\frac{1}{2 \pi} \log \frac{1}{|\zeta|}$ at $a$ and $-\frac{1}{2 \pi}$ $\log \frac{1}{\left|\zeta_{0}\right|}$ at $a_{0}$

Corollary 4. (Maeda [5]) The $\left\{f_{0}^{(a)} \mid a \in R\right\}$-compactification coincides with the Kuramochi compactification.

14. Let $h$ be a function of $D(R)$ and $K$ be a non-polar compact set on $R$. Then, there exists a uniquely determined function $h^{K}$ of class $D(R)$ which is harmonic on $R-K$ and is characterized as

$$
\left\|d h^{K}\right\|=\inf \left\{\left\|d h^{\prime}\right\| \mid h^{\prime}=h \text { quasi-everywhere on } K\right\} .
$$

For any compact set $K^{\prime} \supset K$ we have

$$
h^{K}=\left(h^{K}\right)^{K^{\prime}}=\left(h^{K^{\prime}}\right)^{K}
$$

(Constantinescu-Cornea [2]).

Lemma 7. (Maeda [5]) A function $f \in H D\left(R-K_{0}\right)$ where $K_{0}$ is 
a compact set on $R$, has a generalized normal derivative zero on $\Delta_{D}$, if and only if $f=f^{K}$ for some non-polar compact set $K$ containing $K_{0}$ in its interior.

Let $f_{0}$ be an $L_{0}$-principal function and $\Omega$ be a canonical region carrying all singularities of the $f_{0}$. Let $h_{f_{0}, \Omega}$ be the Dirichlet function which is equal to the $f_{0}$ on $R-\Omega$ and is harmonic on $\Omega$ with boundary value $f_{0}$ on $\partial \Omega$. Then, we get $h_{f_{0}, \Omega}^{K}=h_{f_{0}, \Omega}$ for any compact set $K$ containing $\bar{\Omega}$ by Theorem 4 and Lemma 7 . Because the $h_{f_{0}, \Omega}^{K}$ is harmonic on $R-\Omega=\overline{R-\Omega}$, it is sufficient to assume that $K$ merely contains $\bar{\Omega}$.

Corollary 5. Any $L_{0}$-principal function can be continuously extended to the Kuramochi compactification.

15. Let $\Omega$ be a canonical region and $f$ a real function of class $C^{1}$ on $\partial \Omega$. The principal operator $L_{0}$ associates to $f$ a harmonic function $L_{0} f$ on $R-\bar{\Omega}$. We consider the function

$$
v_{f}=\left\{\begin{array}{lll}
H_{f}^{\Omega} & \text { on } & \bar{\Omega} \\
L_{0} f & \text { on } & R-\bar{\Omega} .
\end{array}\right.
$$

Here $H_{f}^{\Omega}$ denotes the Dirichlet solution in $\Omega$ to the boundary value $f$ on $\partial \Omega$. We can readily verify that the $v_{f}$ has a generalized normal derivative zero on $\Delta_{D}$, and therefore we have $v_{f}^{K}=v_{f}$ for any compact set $K$ containing $\bar{\Omega}$ by Lemma 7 . Considering (14) and (15), we obtain the following characterization of the operator $L_{0}$.

Theorem 6. For a given real function $f$ of class $C^{1}$ on $\partial \Omega$, the function $L_{0} f$ on $R-\bar{\Omega}$ is characterized by the following property; $L_{0} f$ is the function which minimizes Dirichlet integral on $R-\bar{\Omega}$ among those harmonic functions whose boundary value is $f$ on $\partial \Omega$. 
Note. Consider the function

$$
h_{m}=\min \left(m, h^{+}\right)-\min \left(m, h^{-}\right)
$$

for any natural number $m$. Then $h_{m}$ are also Dirichlet functions on $R$, and $\left\|d h_{m}\right\| \leqslant\|d h\|$. By (9) and (9') we obtain

$$
\begin{aligned}
\left.<d u, d h_{m}\right\rangle_{R} & =\lim _{n \rightarrow \infty}\left\langle d u^{(n)}, d h_{m}\right\rangle_{R_{n}} \\
& =\lim _{n \rightarrow \infty}\left(\int_{R^{*}-G} h_{m} d \mu_{n}+\int_{G} h_{m} d \mu_{n}\right) \\
& =\int_{R^{*}} h_{m} d \mu
\end{aligned}
$$

for any $h_{m}$. These convergences are uniform with respect to $m$. Indeed, we have

$$
\begin{gathered}
\left|\int_{R^{*}} h_{m} d \ell-\int_{R^{*}} h_{m} d \mu_{n}\right|=\left|<d\left(u^{(n)}-u\right), d h_{m}>_{R}\right| \\
\leqq\left\|d\left(u^{(n)}-u\right)\right\| \cdot\left\|d h_{m}\right\| \\
\leqq\left\|d\left(u^{(n)}-u\right)\right\| \cdot\|d h\| .
\end{gathered}
$$

The $h$ is summable with respect to the $\mu$ which is seen by (7), and therefore

$$
\int_{R^{*}} h d \mu=\lim _{m \rightarrow \infty} \int_{R^{*}} h_{m} d \mu .
$$

On the other hand, we have

$$
\lim _{m \rightarrow \infty} \int_{R^{*}} h_{m} d \mu_{n}=\int_{R^{*}} h d \mu_{n}
$$

Thus we get

$$
<d u, d h>_{R}=\lim _{n \rightarrow \infty} \int_{R^{*}} h d \mu_{n}=\int_{R^{*}} h d \mu .
$$

KYoto UnIVERSITY

\section{References}

[1] Ahlfors, L. V. and Sario, L.: Riemann Surfaces. Princeton (1960).

[2] Constantinescu, C. und Cornea, A.: Ideale Ränder Riemannscher Flächen. Springer (1963). 
[3] Doob, J. L.: Boundary properties of functions with finite Dirichlet integrals. Ann. Inst. Fourier, 12 (1962), 573-621.

[4] Kusunoki, Y.: Theory of Abelian integrals and its applications to conformal mappings. Mem. Coll. Sci. Univ. Kyoto, Ser. A, Math. 32 (1959), 235-258.

[5] Maeda, F-Y.: Normal derivatives on an ideal boundary. J. Sci. Hiroshima Univ. Ser. A-I, 28 (1964), 113-131.

[6] Nakai, M. and Sario, L.: Construction of Principal functions by orthogonal projection. Canad. J. Math. 18 (1966), 887-896.

[7] Rodin, B. and Sario, L.: Convergence of normal operators. Kódai Math. Sem. Rep. 19 (1967), 165-173.

[8] Sario, L.: A linear operator method on arbitrary Riemann surfaces. Trans. Amer. Math. Soc. 72 (1952), 281-295.

[9] Sario, L. and Oikawa, K.: Capacity functions. Springer (1969).

[10] Watanabe, M.: On a boundary property of principal functions. Pacific J. Math. 31 (1969), 537-545. 